\title{
Chronotaxic systems with separable amplitude and phase dynamics
}

\author{
Yevhen F. Suprunenko, Philip T. Clemson, and Aneta Stefanovska \\ Department of Physics, Lancaster University, Lancaster LAl 4YB, United Kingdom
}

(Received 31 October 2013; published 31 January 2014)

\begin{abstract}
Until recently, deterministic nonautonomous oscillatory systems with stable amplitudes and time-varying frequencies were not recognized as such and have often been mistreated as stochastic. These systems, named chronotaxic, were introduced in Phys. Rev. Lett. 111, 024101 (2013). In contrast to conventional limit cycle models of self-sustained oscillators, these systems posses a time-dependent point attractor or steady state. This allows oscillations with time-varying frequencies to resist perturbations, a phenomenon which is ubiquitous in living systems. In this work a detailed theory of chronotaxic systems is presented, specifically in the case of separable amplitude and phase dynamics. The theory is extended by the introduction of chronotaxic amplitude dynamics. The wide applicability of chronotaxic systems to a range of fields from biological and condensed matter systems to robotics and control theory is discussed.
\end{abstract}

DOI: 10.1103/PhysRevE.89.012922

PACS number(s): 05.45.Xt, 05.90.+m, 89.75.Fb, 05.65.+b

\section{INTRODUCTION}

Real life dynamical systems are often explicitly time dependent: a power-grid network depends on supply and demand [1], the area of a rainforest depends on logging and reforestation [2], a cell must continuously generate ATP to cope with external perturbations $[3,4]$ and the brain continuously matches oxygen and ion supply to its actual function [5] with the recently discovered [6] involvement of astrocytes. In general, every living organism balances its supply and consumption, and even human health depends on the environment [7]. In many cases such time dependences of parameters are oscillatory, and here we argue that this feature should be taken into account explicitly. However, to date time-varying dynamics is typically modeled by an autonomous (i.e., self-contained, not explicitly dependent on time) system of equations. Consequently, systems with nonrepeated, complex, and diverse time-dependent dynamics are often approximated as being chaotic or stochastic. In reality, neither approach captures the characteristic properties of nonautonomous systems, which are far harder to treat because of their explicit time dependence, and their interaction with the external environment.

In mathematics, the theories of nonautonomous dynamical systems [8,9] and random dynamical systems [10,11] have been developed almost in parallel to tackle the problem of systems under external influence. The theories have found practical applications in many fields including climate $[12,13]$, neuroscience $[14,15]$, and evolutionary science [16]. Recently, the theory of tipping points has been developed [17] to explain sudden changes in dynamics in open systems such as the climate. However, the description of oscillatory nonautonomous systems is still often based on conventional autonomous limit cycle models of self-sustained oscillators. In such models the position of a system along a limit cycle, and

Published by the American Physical Society under the terms of the Creative Commons Attribution 3.0 License. Further distribution of this work must maintain attribution to the author(s) and the published article's title, journal citation, and DOI. correspondingly the oscillation itself, can easily be modified by external perturbations: even the smallest perturbation can change the period (frequency) of oscillations. Moreover, the period remains in an autonomous limit cycle model constant in time. Even if it is considered as time dependent, such time-dependent oscillations can still be easily perturbed. In contrast to conventional models, the recently introduced chronotaxic systems [18] exhibit oscillatory nonautonomous dynamics with time-varying frequencies, such that the system resists continuous perturbation by adjusting its frequency and "trying" to make it equal to the unperturbed frequency. For example, a chronotaxic system with a constant oscillatory frequency can have the same frequency when continuously exposed to a relatively weak but constant perturbation, unlike conventional limit cycle models.

In this paper we extend the theory of chronotaxic systems [18] by studying the time-dependent and stable dynamics of both the amplitude and phase of oscillations in the case when these dynamics are separated. This extension is achieved by introducing a time-dependent (driven) steady state or point attractor into the amplitude and phase dynamics. The resulting stable and nonautonomous dynamics is described by drive and response systems which are unidirectionally coupled. The general conditions for the system to be considered chronotaxic are discussed, and these conditions are demonstrated using a theoretical example. Applications of chronotaxic systems to biological systems, condensed matter systems, robotics, and control theory are indicated.

The paper is structured as follows: Sec. II presents a short review and discussion of the theoretical concepts which lead to chronotaxic systems. A generalized model of chronotaxic systems is developed in Sec. III, where a theoretical example is also discussed and the conditions of existence are derived. Applications are discussed in Sec. IV. A summary of results is presented in Sec. V. The Appendix provides an analytical calculation of the time-dependent steady state as a pullback point attractor in a chronotaxic system.

\section{FROM AUTONOMOUS TO NONAUTONOMOUS DYNAMICS AND TIME-DEPENDENT ATTRACTORS}

Chronotaxic systems [18] are based on a unification of two concepts: the concept of self-sustained limit cycle oscillations 


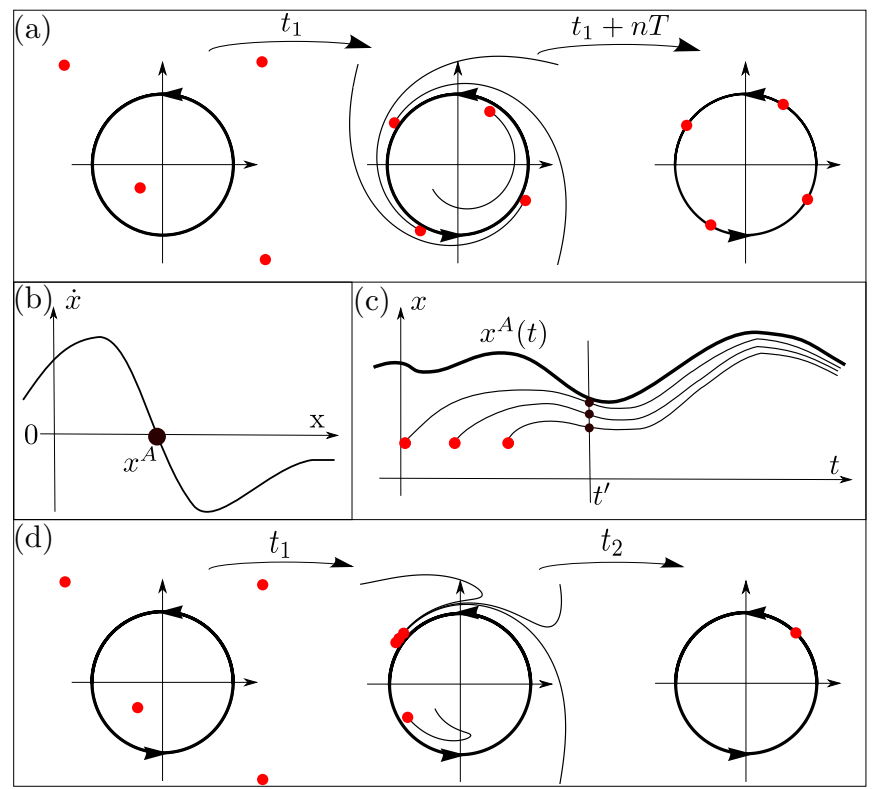

FIG. 1. (Color online) (a) States of a system (red points) do not converge to each other on an autonomous limit cycle (black circle). $T$ is the period of oscillations, and $n$ is a positive integer. (b) A stationary steady state (solid black disk $x^{A}$ ) in a one-dimensional system. (c) Typical dynamics in a system with a time-dependent steady state $x^{A}(t)$. (d) States of a system (red points) converge to each other on a chronotaxic limit cycle (black circle).

[19] and the concept of a time-dependent steady state or a point attractor which can exist only in nonautonomous dynamical systems [8].

The concept of limit cycle oscillators is the most conventional description of self-sustained oscillations within the class of autonomous models. Since their introduction [19] the theory of self-sustained oscillations has been proven to be useful in the description of various phenomena [20,21]. The main property of self-sustained oscillations is that they do not depend on the initial conditions of the system: a system starting from any initial state (allowed within a model) approaches the regime of stable oscillations. Once the stable oscillations are established, they do not decay or change in time. In phase space, such behavior is described by the presence of an asymptotically stable isolated closed curve: a limit cycle $\Gamma_{0}$. All of the system's trajectories in phase space converge to $\Gamma_{0}$.

The motion on the limit cycle has a constant period $T$ : thus if an autonomous dynamical system with a state vector $\mathbf{x} \in R^{m}$ is located on a limit cycle at time $t, \mathbf{x}(t) \in \Gamma_{0}$, then after a period $T$ the system returns to its previous state $\mathbf{x}(t+T)=\mathbf{x}(t)$. Also, it follows that identical limit cycle oscillators with different initial conditions will preserve the distance between each other on a limit cycle, as is shown in Fig. 1(a). This is due to a specific property of a limit cycle: the state space coordinate which is tangent to the limit cycle has a zero Lyapunov exponent, in contrast to all other coordinates which have negative Lyapunov exponents. Such a coordinate is called a phase, denoted as $\alpha$, while the rest of the coordinates with negative Lyapunov exponents are classed as the amplitude. A transformation between an initial state space and an amplitude-phase space in the general case is discussed in Ref. [22]. As a result of a zero Lyapunov exponent, perturbations to the phase neither decay nor grow in time. Consequently, any external perturbations to motion on the limit cycle change the time dependence of the phase $\alpha(t)$ and correspondingly alter the instantaneous frequency of oscillation.

The following example of a limit cycle oscillator, a Poincaré oscillator, in amplitude-phase space $(r, \alpha)$ can be written as

$$
\dot{r}=-\varepsilon_{\Gamma} r\left(r-r_{0}\right), \quad \dot{\alpha}=\omega,
$$

where $\omega=2 \pi / T$ is the frequency of oscillation, and $\varepsilon_{\Gamma}$ and $r_{0}$ are constant. Equations (1) describe a self-sustained oscillator with a limit cycle that is a circle with radius $r_{0}$ and center at the coordinate origin. When the phase $\alpha$ is perturbed, the external perturbations appear on the righthand side of the lower equation in (1). Clearly even the smallest external perturbation, e.g., constant and continuous perturbation, changes the frequency $\dot{\alpha}$ of oscillations. This results from the absence of stability in the phase $\alpha$. The dynamics described by (1) with $\varepsilon_{\Gamma}=1, r_{0}=1, \omega=1.7$ is shown in Fig. 1(a) where $t_{1}=2 \mathrm{~s}, T=2 \pi / 1.7 \mathrm{~s}$.

There is a class of nonautonomous dynamical systems for which stability in the phase $\alpha(t)$ of the oscillations can be maintained while under external perturbation. Nonautonomous dynamical systems are defined by a skew-product flow $[8,23,24]$ generated by the autonomous system of unidirectionally coupled differential equations (which are also called the master-slave configuration [25] or drive and response systems [26]):

$$
\dot{\mathbf{p}}=\mathbf{f}(\mathbf{p}), \quad \dot{\mathbf{x}}=\mathbf{g}(\mathbf{x}, \mathbf{p}),
$$

where $\mathbf{p} \in R^{n}, \mathbf{x} \in R^{m}$, and $\mathbf{f}: R^{n} \rightarrow R^{n}, \mathbf{g}: R^{m} \times R^{n} \rightarrow$ $R^{m}$. Mathematically, a skew product flow is denoted as a pair $(\theta, \varphi)$. Here $\theta$ is a flow, $\theta: R \times P \rightarrow P$, on a base set $P$. The flow $\theta$ corresponds to the first equation in (2). The symbol $\varphi$ denotes a cocycle $\varphi: R \times P \times R^{m} \rightarrow R^{m}$ over $\theta$, such a cocycle is described by the second equation in (2). The cocycle $\varphi$ satisfies the initial value condition, $\varphi(0, \mathbf{p}, \mathbf{x})=\mathbf{x}$, and a cocycle property, $\varphi(t+s, \mathbf{p}, \mathbf{x})=\varphi(t, \theta(s, \mathbf{p}), \varphi(s, \mathbf{p}, \mathbf{x}))$, Ref. [8].

Alternatively the nonautonomous system can be described as a process. Assuming one has a solution $\mathbf{p}(t)$, the dynamics of $\mathbf{x}$ can be expressed in terms of solutions $\mathbf{x}\left(t, t_{0}, \mathbf{x}_{\mathbf{0}}\right)$. Mathematically the process means a continuous mapping

$$
\left(t, t_{0}, \mathbf{x}_{\mathbf{0}}\right) \longmapsto \mathbf{x}\left(t, t_{0}, \mathbf{x}_{\mathbf{0}}\right) \in R^{m}
$$

for all $\left(t, t_{0}, \mathbf{x}_{\mathbf{0}}\right) \in R \times R \times R^{m}$ which satisfy the initial condition, $\mathbf{x}\left(t_{0}, t_{0}, \mathbf{x}_{0}\right)=\mathbf{x}_{0}$, and the cocycle property, $\mathbf{x}\left(t_{2}, t_{0}, \mathbf{x}_{0}\right)=$ $\mathbf{x}\left(t_{2}, t_{1}, \mathbf{x}\left(t_{1}, t_{0}, \mathbf{x}_{0}\right)\right)$.

The subsystems $\mathbf{x}$ and $\mathbf{p}$ can be considered as one system $(\mathbf{p}, \mathbf{x})$, which results in the dynamics of the $\mathbf{x}$ component being nonautonomous in the sense that

$$
\dot{\mathbf{x}}=\mathbf{g}(\mathbf{x}, \mathbf{p}(t)) \text {. }
$$

In this paper the description (4) will be used, and for the time being it will be assumed that the function $\mathbf{p}(t)$ is given.

As identified in Ref. [18], stability of the dynamics in nonautonomous systems can be provided by a driven and therefore time-dependent steady state outside of equilibrium. The driven steady state is considered as a nonautonomous point attractor within the theory of nonautonomous dynamical 
systems [8]. A driven steady state is a generalization of a stationary steady state, whose coordinates all have negative Lyapunov exponents and therefore represent a stable fixed point [Fig. 1(b)]. A time-dependent steady state can exist only in nonautonomous systems. Such a time-dependent steady state or point attractor is denoted by a vector $\mathbf{x}^{A}(t) \in R^{m}$. We consider that the future time dependence of a nonautonomous system is unknown. Therefore it is important to define a steady state at the actual time rather than in the infinite future. Such a state is characterized by so-called pullback attraction [8,27]: all trajectories which start at initial time $t_{0} \in R$ and $\mathbf{x}_{0} \in R^{m}$ are attracted to $\mathbf{x}^{A}(t)$ if one considers the limit $t_{0} \rightarrow-\infty$,

$$
\lim _{t_{0} \rightarrow-\infty} \mathbf{x}\left(t, t_{0}, \mathbf{x}_{0}\right)=\mathbf{x}^{A}(t)
$$

The steady state also attracts system states when time goes to infinity. Thus a steady state is also a forward point attractor:

$$
\lim _{t \rightarrow \infty} \mathbf{x}\left(t, t_{0}, \mathbf{x}_{0}\right)=\mathbf{x}^{A}(t) .
$$

The point $\mathbf{x}^{A}(t)$ should also satisfy the condition of invariance

$$
\mathbf{x}\left(t, t_{0}, \mathbf{x}^{A}\left(t_{0}\right)\right)=\mathbf{x}^{A}(t) .
$$

The typical dynamics starting from different initial conditions in a system with a time-dependent steady state $x^{A}(t)$ is shown in Fig. 1(c).

Concepts discussed in this section can be used for the description of oscillatory systems with time-varying and stable frequencies. These oscillatory systems can resist external fluctuations by adjusting themselves to be maximally close to their unperturbed state. It is important to stress that nonautonomous systems with time-dependent steady states or point attractors represent an appropriate model [18] which describes such systems. Previously, the absence of this model led to attempts to describe deterministic nonautonomous oscillatory systems with time-varying and stable frequencies as, for example, stochastic.

In Ref. [18] we identified which nonautonomous systems with time-dependent steady states represent an adequate description of time-dependent and stable oscillatory dynamics. These systems were recognized as a separate class of nonautonomous dynamical systems. In order to stress that their dynamics is ordered in time and therefore resists continuous perturbation, these systems were named chronotaxic ( chronos $=$ time, taxis $=$ order).

In the next section the detailed definition and properties of chronotaxic systems with separable amplitude and phase dynamics are presented. In this respect it is not only the phase that is considered to be time-dependent and stable but also the amplitude dynamics.

\section{CHRONOTAXIC SYSTEMS}

\section{A. Model of separable chronotaxic phase and amplitude dynamics}

Consider a conventional model of an autonomous dynamical system which admits a limit cycle $\Gamma_{0}$. In the general case of a high dimensional system, suitable coordinates can be chosen: one coordinate, a phase, describes the motion along the limit cycle, while other coordinates describe the dynamics in perpendicular directions to the limit cycle. In contrast to autonomous limit cycle oscillators, where the phase and frequency can easily be perturbed, we require that the phase and frequency of oscillations in chronotaxic systems cannot easily be changed by external perturbations. Thus, it is required that the phase has a time-dependent steady state or a point attractor. Additionally, it is assumed this is achieved without influencing the amplitude dynamics. This means that the phase and amplitude dynamics must be separable. For the sake of simplicity, consider a two-dimensional (2D) system and assume it can be transformed to polar coordinates where the angular motion corresponds to phase dynamics, and the radial component determines the amplitude of oscillation. In such a case the limit cycle is a circle. The amplitude and phase dynamics are described similarly to Eq. (4):

$$
\dot{\alpha}_{\mathbf{x}}=g_{\alpha}\left(e^{i \alpha_{\mathbf{x}}}, \mathbf{p}(t)\right) \quad \dot{r}_{\mathbf{x}}=g_{r}\left(r_{\mathbf{x}}, \mathbf{p}(t)\right)
$$

where $\alpha_{\mathbf{x}}$ is the phase $\alpha_{\mathbf{x}} \in(-\infty,+\infty), r_{\mathbf{x}} \in(0,+\infty)$, $g_{\alpha}: S^{1} \times R^{n} \rightarrow R$, and $g_{r}: R_{+} \times R^{n} \rightarrow R$. The value of the phase increases by $2 \pi$ after each full cycle along $\Gamma_{0}$. The phase wrapped on the circle is pullback and forward attracted to a point attractor or steady state which is characterized by a phase $\alpha_{\mathbf{x}}^{A}(t) \in R$ :

$$
\begin{gathered}
\lim _{t_{0} \rightarrow-\infty} \operatorname{Re}\left(e^{i \alpha_{\mathbf{x}}\left(t, t_{0}, \alpha_{0}\right)}-e^{i \alpha_{\mathbf{x}}^{A}(t)}\right)=0, \\
\lim _{t \rightarrow \infty} \operatorname{Re}\left(e^{i \alpha_{\mathbf{x}}\left(t, t_{0}, \alpha_{0}\right)}-e^{i \alpha_{\mathbf{x}}^{A}(t)}\right)=0 .
\end{gathered}
$$

Correspondingly, the unwrapped phase is attracted to one of the values $\alpha_{\mathbf{x}}^{A}(t) \pm 2 \pi k$, where $k$ is an arbitrary integer number. The condition of invariance can be rewritten as

$$
g_{\alpha}\left(e^{i \alpha_{\mathbf{x}}^{A}(t)}, \mathbf{p}(t)\right)=\dot{\alpha}_{\mathbf{x}}^{A}(t) .
$$

This means that the point attractor exists only if there is such a phase $\alpha_{\mathbf{x}}^{*}(t)$ which moves with certain velocity $v(t)=\dot{\alpha}_{\mathbf{x}}^{*}(t)$, and at any moment of time $t$ the value of the function $g_{\alpha}$ at this phase is equal to the velocity $v(t)$. A negative Lyapunov exponent of the phase corresponds to the following condition:

$$
\left.\frac{\partial}{\partial \alpha_{\mathbf{x}}} g_{\alpha}\left(e^{i \alpha_{\mathbf{x}}}, \mathbf{p}(t)\right)\right|_{\alpha_{\mathbf{x}}=\alpha_{\mathbf{x}}^{A}(t)}<0 .
$$

The point attractor should therefore be located at the phase interval where the function $g_{\alpha}$ has a negative slope at the given moment of time. From (11) and (12) it follows that there should be such a rotating reference frame where the point attractor appears as a stationary and stable fixed point.

In the case of autonomous and separable amplitude and phase dynamics, the presence of a limit cycle is equivalent to the presence of a stable fixed point in radial dynamics. We extend this notion by considering the system as nonautonomous, and therefore by characterizing the amplitude dynamics by a time-dependent point attractor $r_{\mathbf{x}}^{A}(t)$. This corresponds to an attractive cycle $\Gamma_{0}(t)$ with a varying radius. The attractor $r_{\mathbf{x}}^{A}(t)$ should satisfy conditions similar to Eqs. (9)-(12):

$$
\begin{gathered}
\lim _{t_{0} \rightarrow-\infty}\left[r_{\mathbf{x}}\left(t, t_{0}, r_{0}\right)-r_{\mathbf{x}}^{A}(t)\right]=0, \\
\lim _{t \rightarrow \infty}\left[r_{\mathbf{x}}\left(t, t_{0}, r_{0}\right)-r_{\mathbf{x}}^{A}(t)\right]=0, \\
g_{r}\left(r_{\mathbf{x}}^{A}(t), \mathbf{p}(t)\right)=\dot{r}_{\mathbf{x}}^{A}(t), \\
\left.\frac{\partial}{\partial r_{\mathbf{x}}} g_{r}\left(r_{\mathbf{x}}, \mathbf{p}(t)\right)\right|_{r_{\mathbf{x}}=r_{\mathbf{x}}^{A}(t)}<0 .
\end{gathered}
$$


Thus, in order to describe a chronotaxic system, Eqs. (8) should have a unique time-dependent steady state or point attractor $\mathbf{x}^{A}(t)$,

$$
\mathbf{x}^{A}(t)=\left(r_{\mathbf{x}}^{A} \cos \left(\alpha_{\mathbf{x}}^{A}\right), r_{\mathbf{x}}^{A} \sin \left(\alpha_{\mathbf{x}}^{A}\right)\right),
$$

where $r_{\mathbf{x}}^{A}(t)$ and $\alpha_{\mathbf{x}}^{A}(t)$ satisfy conditions (9)-(16).

We stress that Eqs. (2) and (4) represent an unperturbed chronotaxic system. Therefore the system p in Eq. (2) includes only that part of the environment external to $\mathbf{x}$ which generates a stable nonautonomous dynamics in $\mathbf{x}$. The influences of the environment external to $\mathbf{x}$ and $\mathbf{p}$ are considered as perturbations.

As follows from conditions (9)-(16), the unperturbed chronotaxic system has a time-dependent point attractor or steady state at all times. The presence of such a state implies that the system is asymptotically stable [i.e., $\forall \mathbf{x}_{01}, \mathbf{x}_{02}$ : $\left.\lim _{t \rightarrow \infty}\left\|\mathbf{x}\left(t, t_{0}, \mathbf{x}_{01}\right)-\mathbf{x}\left(t, t_{0}, \mathbf{x}_{02}\right)\right\|=0\right]$. For a discussion of asymptotically stable systems see Refs. [26,28]. However, not all asymptotically stable systems have point attractors or steady states at all times (before the limit $t \rightarrow \infty$ is achieved) because the additional conditions, the existence of a pullback attractor (5) and its invariance (7), are not necessarily satisfied by asymptotically stable systems. Therefore chronotaxic systems are a subclass of asymptotically stable systems.

Also, the unidirectionally coupled systems (2), where the driven system is asymptotically stable, were shown to lead to general synchronization [26] in that as time goes to infinity, the states of the drive system $\mathbf{p},\left(\mathbf{p} \in R^{n}\right)$, and response system $\mathbf{x}$, $\left(\mathbf{x} \in R^{m}\right)$, become connected by a (static) functional relation $\mathbf{H}: R^{n} \rightarrow R^{m}, \mathbf{x}=\mathbf{H}(\mathbf{p})$. Because of the asymptotic stability of the unperturbed chronotaxic system $\mathbf{x}$, the $\mathbf{p}$ and $\mathbf{x}$ systems are generally synchronized. However, from the previous paragraph it follows that chronotaxic systems represent a special subclass of generally synchronized systems, because not all generally synchronized systems have time-dependent steady states at all times.

One of the most important messages in this work and in Ref. [18], therefore, is that it is not the limit $t \rightarrow \infty$ that should be considered but the actual time $t$. The system and its properties are to be studied at this actual time $t$. It is especially important for practical applications and studies of real systems where the limit $t \rightarrow \infty$ cannot be achieved. This constitutes the importance and special place of nonautonomous and particularly chronotaxic systems.

Stability in the dynamics of a response system was also achieved in replica synchronized systems, e.g., in chaotic unidirectionally coupled systems [28-30] (see also Ref. [21]). After some synchronization transient has elapsed, the response systems in $[28,29]$ can be considered as particular and interesting examples of chronotaxic systems. Systems that exhibit "replica synchronization" represent a very particular example of a chronotaxic system. Chronotaxic systems can be realized in various ways beyond "replica synchronization," and the drive and response systems can be totally different dynamical systems with different dimensions. We also specify that the chronotaxic system $\mathbf{x}$ is not chaotic because of the presence of a time-dependent steady state and consequent insensitivity to initial conditions. Nevertheless if drive system $\mathbf{p}$ has chaotic dynamics, the dynamics of a steady state $\mathbf{x}^{A}(t)$ may also look chaotic, e.g., as in Ref. [29].

For the sake of simplicity consider a particular example of a chronotaxic system which is generated by two unidirectionally coupled similar or identical systems. These coupled systems can be relevant to many applications. A description of the dynamics of the phase $\alpha_{\mathbf{x}}(t)$, which has an attractor $\alpha_{\mathbf{x}}^{A}(t)$, can be expressed by the coupling of $\alpha_{\mathbf{x}}$ to a single time-dependent phase $\alpha_{\mathbf{p}}(t)$ :

$$
\dot{\alpha}_{\mathbf{x}}=g_{\alpha}\left(e^{i \alpha_{\mathbf{x}}}, e^{i \alpha_{\mathbf{p}}(t)}\right) .
$$

Here $g_{\alpha}: S^{1} \times S^{1} \rightarrow R$, and $\alpha_{\mathbf{p}} \in R$ has an angular velocity $\dot{\alpha}_{\mathbf{p}}(t)$. We assume that the motion of the attractor $\alpha_{\mathbf{x}}^{A}(t)$ is completely defined by the function $\alpha_{\mathbf{p}}(t)$ and by a coupling function $g_{\alpha}$. It is important that phase slips between $\alpha_{\mathbf{p}}$ and $\alpha_{\mathbf{x}}$ do not occur; i.e., the difference $\left|\alpha_{\mathbf{p}}-\alpha_{\mathbf{x}}\right|$ lies within a constant interval of width $2 \pi$. Correspondingly, the description of the amplitude dynamics can be made via the coupling of $r$ to an amplitude $r_{\mathbf{p}}(t)$,

$$
\dot{r}_{\mathbf{x}}=g_{r}\left(r_{\mathbf{x}}, r_{\mathbf{p}}(t)\right),
$$

where $g_{r}: R_{+} \times R_{+} \rightarrow R$.

As an example of a $2 \mathrm{D}$ chronotaxic system, consider a system which is described by a vector $\mathbf{x}=\left(r_{\mathbf{x}} \cos \left(\alpha_{\mathbf{x}}\right), r_{\mathbf{x}} \sin \left(\alpha_{\mathbf{x}}\right)\right)$ and by the following equations:

$$
\begin{gathered}
\dot{r}_{\mathbf{x}}=\varepsilon_{\Gamma} r_{\mathbf{x}}\left[r_{\mathbf{p}}(t)-r_{\mathbf{x}}\right], \\
\dot{\alpha}_{\mathbf{x}}=-\varepsilon_{A} \sin \left[\alpha_{\mathbf{x}}-\alpha_{\mathbf{p}}(t)\right] .
\end{gathered}
$$

Here $\varepsilon_{\Gamma}=3$ and $\varepsilon_{A}=7$. We assume the following functions $r_{\mathbf{p}}(t)$ and $\alpha_{\mathbf{p}}(t)$ :

$$
\begin{aligned}
& r_{\mathbf{p}}(t)=\left\{\begin{array}{c}
1.3, t<0 ; \\
0.5,0 \leqslant t<0.5 ; \\
0.5+0.16(t-0.5)+0.1(t-0.5)^{2}, t>0.5 .
\end{array}\right. \\
& \alpha_{\mathbf{p}}(t)= \begin{cases}t, & t<0 ; \\
2 \pi\left(0.7 t-0.4 \omega_{1}^{-1} \sin \left(\omega_{1} t\right)\right), & t \geqslant 0,\end{cases}
\end{aligned}
$$

where $\omega_{1}=1.375 \pi$. Later it will be shown that the functions (22) and (23) generate chronotaxic dynamics in the response system. When $t<0$, the steady state of the system considered can be found analytically as a synchronized state: $\alpha_{\mathbf{x}}^{A}(t<0)=$ $\alpha_{\mathbf{p}}(t)-\arcsin \left(1 / \varepsilon_{A}\right), r_{\mathbf{x}}^{A}(t<0)=r_{\mathbf{p}}(t)$. Using the conditions of invariance (11) and (15), the time-dependent steady state can be found in the interval $t \geqslant 0$ by integrating the corresponding dynamical equations. The coordinates of the drive system influence the coordinates of the time-dependent steady state in a response system, but they are not the same, as is shown in Figs. 2(a) and 2(b).

Equations (20) and (21) are integrated for different initial conditions. The resulting dynamics of the phase and amplitude, and their combined dynamics, are shown in Figs. 3(a), 3(b), and 3(c), respectively. States of the system starting from different initial conditions in Fig. 3(a) can originate from a time-dependent steady state which receives different positive increments to its phase and different changes to its amplitude because of perturbations. As a result, a chronotaxic system can return to the steady state either without a phase slip, e.g., as the 

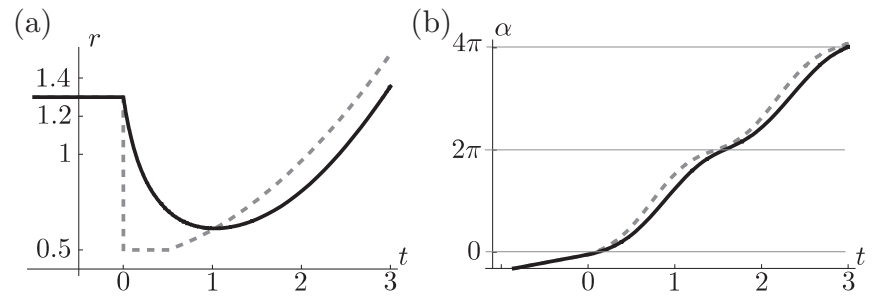

FIG. 2. (a) Dynamics of $r_{\mathbf{p}}(t)$ (gray dashed line) and $r_{\mathbf{x}}^{A}(t)$ (black line) from Eqs. (20) and (22). (b) Dynamics of $\alpha_{\mathbf{p}}(t)$ (gray dashed line) and $\alpha_{\mathbf{x}}^{A}(t)$ (black line) from Eqs. (21) and (23).

thin-solid, dash-dotted, thin-dashed, and dotted lines, or with a phase slip, e.g., as a thick dashed line. This means that the system is able to sustain its dynamics against perturbations. During these processes the instantaneous frequency of the system can change sign, as in thin-solid, dash-dotted, thindashed, and dotted trajectories. One can also see that two closely located perturbed states of a system can temporarily diverge before converging to the steady state, as shown by thin-solid and dash-dotted lines or by thick-dashed and thin-dashed lines in Fig. 3(a). Similar temporary divergence is possible in the amplitude dynamics as shown in Fig. 3(b) by the dotted trajectory which temporarily diverge from a time-dependent steady state $r_{\mathbf{x}}^{A}(t)$.

Taking into account the various possible behaviours mentioned above, the observed dynamics of chronotaxic systems may look very complex. Such complexity can be generated by the motion of a time-dependent steady state, shown as a solid black disk in Fig. 3(c), as well as by perturbations and consequent temporary divergent trajectories and phase slips. Under such perturbations the stability evident in the unper- turbed chronotaxic limit cycle may be lost. Yet, chronotaxic systems are in general able to retain their stability through the time-dependent adjustments of the position of a driven steady state and movement of a chronotaxic limit cycle. This means that complex yet deterministic dynamics can be generated. Patterns of behavior can therefore be identified and linked to the states of the system, which can be distinguished from each other, for example a healthy heart from a diseased heart.

We now summarize the properties of chronotaxic systems with separable phase and amplitude dynamics. These systems are nonautonomous and oscillatory and have a time-varying and stable amplitudes due to a time-varying steady state in the amplitude dynamics. Additionally, the frequency of the oscillations is stable against external perturbations due to a time-dependent steady state in the phase dynamics. External perturbations cause the system to deviate from its driven steady state. Despite this, the frequency and amplitude resist external perturbations and therefore cannot be easily perturbed. The system therefore maintains its initial unperturbed dynamics. Physically, the stable amplitude and phase dynamics mean that the system has to be thermodynamically open and it should also have an internal source of energy to overcome dissipations, similarly to self-sustained oscillators.

\section{B. Conditions of existence}

We now discuss some theoretical examples using the definition of a chronotaxic system in the case of separable phase and amplitude dynamics (9)-(16), together with assumptions (18) and (19). Our aim is to find the corresponding conditions on the dynamics of $\alpha_{\mathbf{p}}(t)$ and $r_{\mathbf{p}}(t)$.
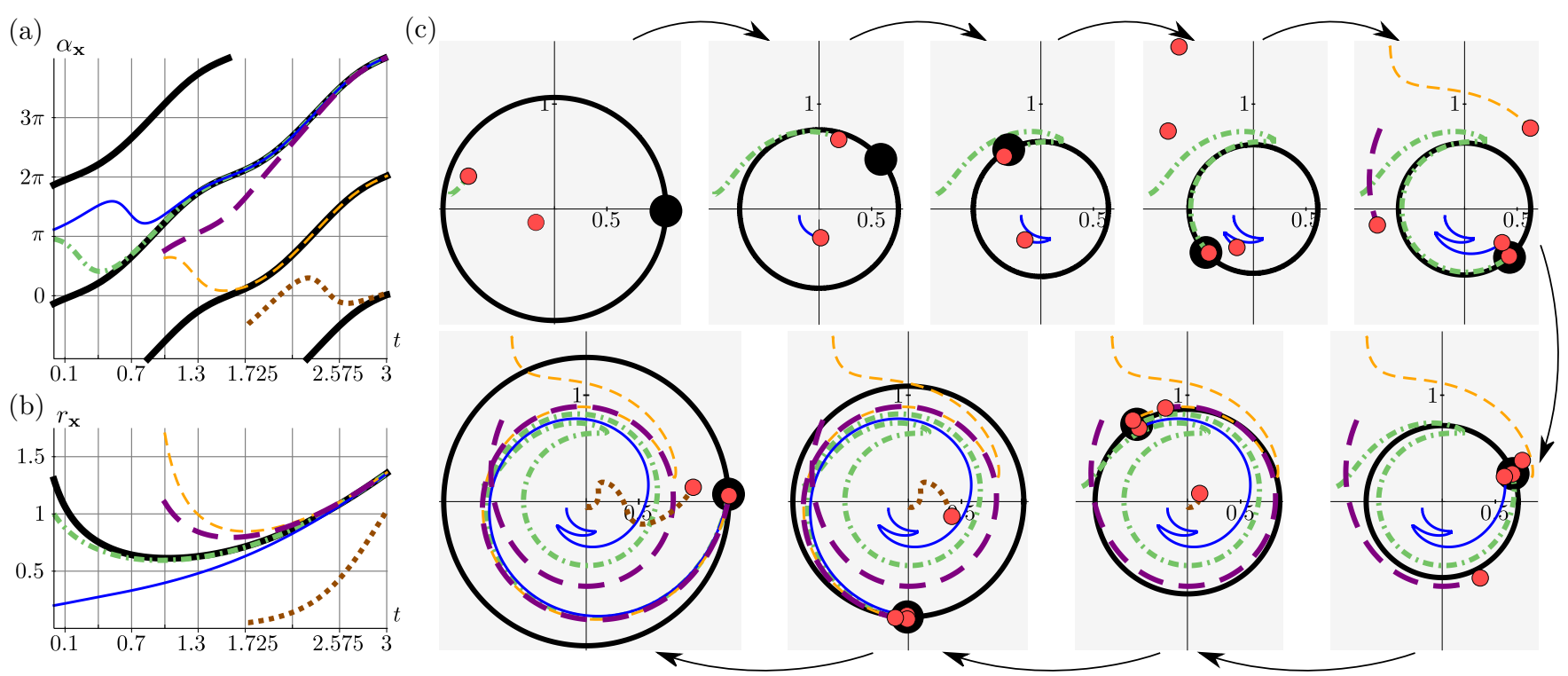

FIG. 3. (Color online) Chronotaxic dynamics. Different trajectories are indicated by the same colors and structure throughout, in particular by thin-solid line (blue), dash-dotted line (green), thick-dashed line (purple), thin-dashed line (orange), and dotted (brown) line. (a) Chronotaxic phase dynamics given by Eqs. (21) and (23). Black thick lines correspond to time-dependent steady states $\alpha_{\mathbf{x}}^{A}(t)+2 \pi k, k=-1,0,1,2$. (b) Chronotaxic amplitude dynamics given by Eqs. (20) and (22), where the black thick line denotes $r_{\mathbf{x}}^{A}(t)$. (c) Total chronotaxic dynamics of a system (20)-(23). The black circle denotes $\Gamma_{0}(t)$ while the solid black disk denotes a time-dependent steady state with polar coordinates $r_{\mathbf{x}}^{A}(t)$ and $\alpha_{\mathbf{x}}^{A}(t)$. States of the system are shown by red (gray) small disks. The small arrows denote time intervals equal to $0.3 \mathrm{~s}$, whereas the larger arrows denote intervals of $0.425 \mathrm{~s}$. 

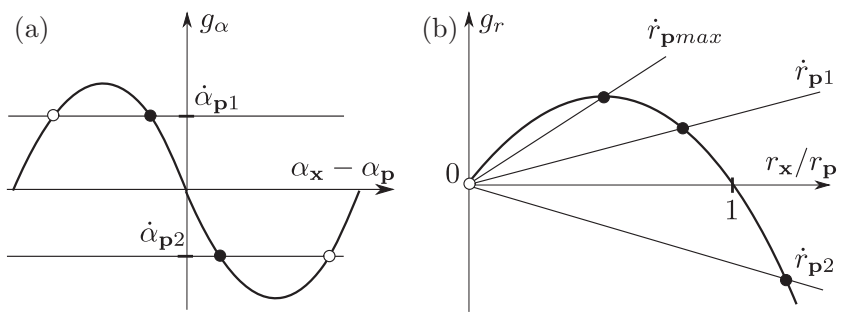

FIG. 4. Conditions of existence of chronotaxic phase and amplitude dynamics. (a) Illustration of Eq. (25) for the chronotaxic system given by Eqs. (18) and (24). (b) Illustration of Eqs. (29) and (30).

\section{Phase dynamics}

Consider a chronotaxic system with $g_{\alpha}$ given by

$$
g_{\alpha}\left(e^{i \alpha_{\mathbf{x}}}, e^{i \alpha_{\mathbf{p}}}\right)=-\varepsilon_{A}(t) \sin \left[\alpha_{\mathbf{x}}-\alpha_{\mathbf{p}}(t)\right]+\omega_{0}+\xi_{\alpha}(t) .
$$

First assume that the parameters in (24), $\varepsilon_{A}(t), \dot{\alpha}_{\mathbf{p}}(t), \xi_{\alpha}(t)$, are constant in time, and that $\varepsilon_{A}(t)$ is always of the same sign. A time-dependent point attractor, according to the condition (12), belongs to the interval where the function $g_{\alpha}$ has a negative slope at a given moment of time. Any phase within this interval moves with a velocity $\dot{\alpha}_{\mathbf{p}}$, and the condition (12) is satisfied only if the value of $g_{\alpha}$ in this interval can be equal to $\dot{\alpha}_{\mathbf{p}}$, i.e., if

$$
\min \left[g_{\alpha}\right]<\dot{\alpha}_{\mathbf{p}}<\max \left[g_{\alpha}\right] ;
$$

see Fig. 4(a). Using Eq. (24), Eq. (25) is rewritten as

$$
\left|\dot{\alpha}_{\mathbf{p}}-\omega_{0}-\xi_{\alpha}\right|<\left|\varepsilon_{A}\right| .
$$

The same result can be obtained as a condition of existence for a stable fixed point in the rotating reference frame with $\psi=\alpha_{\mathbf{x}}-\alpha_{\mathbf{p}}(t)$. Equation (26) constitutes the Arnold tongue: the area of synchronization of two unidirectionally coupled oscillators with phases $\alpha_{\mathbf{p}}$ and $\alpha_{\mathbf{x}}$ and frequencies $\dot{\alpha}_{\mathbf{p}}$ and $\omega_{0}$ with an effective coupling strength $\varepsilon_{A}$. At the border where the condition (26) is violated a saddle-node bifurcation occurs. When $\dot{\alpha}_{\mathbf{p}}$ and $\xi_{\alpha}$ are constant the point attractor can be found analytically, $\alpha_{\mathbf{x}}^{A}(t)=\alpha_{\mathbf{p}}(t)-\arcsin \left[\left(\dot{\alpha}_{\mathbf{p}}-\omega_{0}-\xi_{\alpha}\right) / \varepsilon_{A}\right]$.

Assuming a time-dependent steady state at some initial time, consider changes in parameters for which (26) is satisfied. In such cases an assumed time-dependent steady state will not move to the area with a positive slope in function $g_{\alpha}$. Therefore, the local stability condition (12) will be still satisfied, the invariance condition (11) will be preserved, and all points will continue to converge to a point attractor as in (9)-(10). Thus, if time-dependent parameters are considered, the point attractor for a phase exists at any instance of time if the condition (26) is satisfied at that instant of time and if the coupling strength $\varepsilon_{A}(t)$ does not change its sign:

$$
\left|\dot{\alpha}_{\mathbf{p}}(t)-\omega_{0}(t)-\xi_{\alpha}(t)\right|<\left|\varepsilon_{A}(t)\right| .
$$

It is easy to check that the example given by (21) and (23) satisfies (27).

\section{Amplitude dynamics}

Consider the amplitude dynamics (19) with

$$
g_{r}\left(r_{\mathbf{x}}, r_{\mathbf{p}}(t)\right)=-\varepsilon_{\Gamma}(t) r_{\mathbf{x}}\left[r_{\mathbf{x}}-r_{\mathbf{p}}(t)\right] .
$$

By taking the parameters $\varepsilon_{\Gamma}$ and $r_{\mathbf{p}}$ to be constant in time, the point attractor $r_{\mathbf{x}}^{A}$ always exists when $\varepsilon_{\Gamma}>0$ and $r_{\mathbf{p}} \geqslant$ 0 . Assuming time-dependent parameters one can see that variations of $\varepsilon_{\Gamma}(t)$ [as long as $\varepsilon_{\Gamma}(t)>0$ and $r_{\mathbf{p}}(t)$ is constant] will not lead to a bifurcation, and a point attractor $r_{\mathbf{x}}^{A}(t)$ will exist. However, certain changes of $r_{\mathbf{p}}(t)$ may move the point attractor to a region with a positive derivative of $g_{r}$ on $r_{\mathbf{x}}$, and therefore the point attractor will no longer exist according to the condition (16). In order to have a time-dependent steady state, the condition (15) should be satisfied. For the model considered the velocity $\dot{r}$ of the point which corresponds to a given value $g_{r}\left(r_{\mathbf{x}}, r_{\mathbf{p}}(t)\right)$ at time $t$ is given by $\dot{r}_{\mathbf{p}} r_{\mathbf{x}} / r_{\mathbf{p}}$. It should be equal to a function $g_{r}\left(r_{\mathbf{x}}, r_{\mathbf{p}}(t)\right)$ according to (15). Substituting $g_{r}$ from (28) one obtains

$$
-\varepsilon_{\Gamma}(t) r_{\mathbf{x}}\left[r_{\mathbf{x}}-r_{\mathbf{p}}(t)\right]=\dot{r}_{\mathbf{p}}(t) \frac{r_{\mathbf{x}}}{r_{\mathbf{p}}(t)} .
$$

Graphically this equation is shown in Fig. 4(b). Substituting $r_{\mathbf{x}}$ from the interval $\left(r_{\mathbf{p}} / 2,+\infty\right)$ where the condition (16) can be satisfied, one obtains that a point attractor $r_{\mathbf{x}}^{A}(t)$ exists if, at any moment of time $t$, the following condition holds:

$$
\dot{r}_{\mathbf{p}}(t)<\dot{r}_{\mathbf{p m a x}}=\varepsilon_{\Gamma}(t) r_{\mathbf{p}}^{2}(t) / 2 .
$$

When $\dot{r}_{\mathbf{p}}(t)=c \varepsilon_{\Gamma}(t) r_{\mathbf{p}}^{2}(t)$, where $c$ is a constant and $0<c<$ $1 / 2$, the solution of Eq. (29) satisfies conditions (10)-(12). It therefore determines a time-dependent steady state: $r_{\mathbf{x}}^{A}(t)=$ $(1-c) r_{\mathbf{p}}(t)$. However, in such a case the basin of attraction is given only by $r_{\mathbf{x}} \in\left(r_{\mathbf{p}}(t) / 2,+\infty\right)$, no points with $r_{\mathbf{x}}<r_{\mathbf{p}}(t) / 2$ will reach the steady state $r_{\mathbf{x}}^{A}(t)$. When $\dot{r}_{\mathbf{p}}(t)>\dot{r}_{\text {pmax }}$ no steady state exists in the system. At the point $r_{\mathbf{p}}(t)=0$ a transcritical bifurcation occurs and a point $r_{\mathbf{x}}=0$ changes its stability from being repulsive to attractive and therefore becomes a stationary steady state.

As one can see, (30) is satisfied by the system given in (20) and (22). Moreover, the possibility of having large negative velocities $\dot{r}_{\mathbf{p}}$ allows us to consider a jumplike decrease in the value of $r_{\mathbf{p}}$ which can be interpreted as a switching between different drive systems.

\section{APPLICATIONS AND DISCUSSIONS}

Advances in the inverse approach (which studies properties of a system from the information contained in its observable variables) have made it possible to observe similar timedependent dynamics in very different systems, such as the cardiovascular system [31], mitochondrial oscillations [32], the brain $[33,34]$, and surface state electrons on liquid helium [35]. The variety of these systems suggests that the common features of their dynamics are generated from a universal basis. These systems are oscillatory and have stable amplitudes; therefore the conventional model of self-sustained limit-cycle oscillators [19] is able to model this part of the observed features. For example, the model [36] of the cardiovascular system as five coupled self-sustained autonomous limit cycle oscillators reproduces the main characteristic features of the observed cardio-respiratory interactions. However, it was shown $[37,38]$ that an explanation of the variability of cardiac and respiratory frequencies within the model [36] requires consideration of the effect of noise [37] or for the system to be near the onset of oscillation death [38]. In contrast to this, frequencies of oscillations in the systems [31-35], 
despite being externally perturbed, appear to have stable patterns in time that are resistant to external perturbations. This leads to the inference that these characteristic frequencies are time-varying and dynamically stable. This important feature of the observed dynamics [31-35] cannot be explained by conventional models, but can be described by the model of chronotaxic systems introduced in Ref. [18] and presented in details and extended in this paper.

The applicability of chronotaxic systems to living systems is also supported by the general model of biological systems introduced by Friston [34] to describe self-organizing behavior in biological systems. Thus, on the assumption that the external perturbations of the dynamics of the drive system are suppressed, the drive system $\mathbf{p}$ resembles inner variables in Friston's model [34]. According to Ref. [34] these inner variables cannot be perturbed by the external environment. Simultaneously, the response system $\mathbf{x}$ from Eq. (2) resembles the external variables which are exposed to external perturbation in the same model [34]. However, in contrast to Ref. [34], the model of chronotaxic system [18] is not based on any assumptions about the external perturbations, but instead it employs the idea of a time-dependent steady state of a nonautonomous dynamical system.

Driven (time-dependent) steady states are also actively studied in solid state physics [39] and quantum critical systems [40]. In these areas a nontrivial out-of-equilibrium universality of a driven steady state was obtained [40] and the possibility of engineering new properties of a system, e.g., graphene, by driving it to the nonequilibrium steady state was demonstrated [39]. Chronotaxic systems, based on the idea of a time-dependent steady state, can potentially be used to describe phenomena in these fields in terms of the theory of dynamical systems.

Practical applications stimulate the introduction to the inverse approach of a theory of chronotaxic systems. The need to characterize a system as nonautonomous resulted in the generation of methods which provide time-localized analysis of dynamical systems. In the studies of cardiovascular and brain dynamics, the wavelet transform was applied to cope with the inherent time variability of such systems [41] and other wavelet-based methods $[42,43]$, phase coherence methods $[43,44]$, and most recently Bayesian-based inference methods $[45,46]$ have since been introduced to cope with the nonautonomicity. Further development is now needed in the inverse approach to chronotaxic systems, which is now tackled in Ref. [47].

In robotics and control problems it may sometimes be necessary to construct an oscillatory system having a timedependent steady state with given properties and dynamics. The design of oscillators with properties needed was discussed in Ref. [22], although the oscillators were considered as autonomous and the stability properties were not discussed there. The analytically solvable examples of chronotaxic systems allow for the construction of an oscillator with a prescribed time-dependent steady state. The description of phase dynamics with a given time-dependent steady state described by a phase $\alpha_{\mathbf{x}}^{A}(t)$ can be done, for example, by the following simple model:

$$
\dot{\alpha}_{\mathbf{x}}=\dot{\alpha}_{\mathbf{x}}^{A}(t)-\varepsilon(t) \sin \left[\alpha_{\mathbf{x}}-\alpha_{\mathbf{x}}^{A}(t)\right],
$$

where $\varepsilon(t)>0$, or by the model

$$
\dot{\alpha}_{\mathbf{x}}=\varepsilon \dot{\alpha}_{\mathbf{x}}^{A}(t) \sin \left[\alpha_{\mathbf{x}}^{A}(t)-\alpha_{\mathbf{x}}+\arcsin \frac{1}{\varepsilon}\right],
$$

where $\varepsilon \geq 1$ and the angular velocity $\dot{\alpha}_{\mathbf{x}}^{A}(t)$ is always positive and is not equal to zero [the derivation of a model (32) is presented in the Appendix]. Equations (31) and (32) both allow the control of a time-dependent relaxation time $\tau\left(t, t_{0}\right)$ which describes the phase dynamics when $\left|\alpha_{\mathbf{x}}-\alpha_{\mathbf{x}}^{A}(t)\right| \ll 1$,

$$
\alpha_{\mathbf{x}}-\alpha_{\mathbf{x}}^{A}(t) \approx \alpha_{0} e^{-\left(t-t_{0}\right) / \tau\left(t, t_{0}\right)} .
$$

In the case of Eq. (31),

$$
\frac{1}{\tau\left(t, t_{0}\right)}=\frac{1}{\left(t-t_{0}\right)} \int_{t_{0}}^{t} \varepsilon\left(t^{\prime}\right) d t^{\prime},
$$

and for Eq. (32),

$$
\frac{1}{\tau\left(t, t_{0}\right)}=\frac{\sqrt{\varepsilon^{2}-1}}{\left(t-t_{0}\right)} \int_{t_{0}}^{t} \dot{\alpha}_{\mathbf{x}}^{A}\left(t^{\prime}\right) d t^{\prime} .
$$

For amplitude dynamics the analytical solution can be found near the limit cycle. In such cases the dynamics can be approximated by a model which neglects the unstable fixed point in the center of the limit cycle, e.g., as in the following equation:

$$
\dot{r}_{\mathbf{x}}=-\varepsilon(t)\left[r_{\mathbf{x}}-r_{\mathbf{p}}(t)\right], \quad r_{\mathbf{x}}>0, r_{\mathbf{p}}(t)>0,
$$

with the assumption $\left|r_{\mathbf{x}}-r_{\mathbf{p}}(t)\right| \ll r_{\mathbf{p}}(t)$. In terms of a timedependent steady state described by $r_{\mathbf{x}}^{A}(t)$, Eq. (36) can be rewritten as

$$
\dot{r}_{\mathbf{x}}=-\varepsilon(t)\left[r_{\mathbf{x}}-r_{\mathbf{x}}^{A}(t)\right]+\dot{r}_{\mathbf{x}}^{A}(t)
$$

Its solution is analogous to Eq. (33) with the inverse relaxation time $\tau^{-1}$ given by Eq. (34).

The variables $\alpha_{\mathbf{x}}^{A}(t)$ or $r_{\mathbf{x}}^{A}(t)$ represent steady states in Eqs. (31)-(37) by construction, and hence there are no constraints on their velocities.

These applications to robotics and control problems, in addition to those mentioned previously, are used here to illustrate the applicability of the new class of systems spanning a vast range from the natural to the manmade. These particular examples probably just represent the tip of the iceberg in terms of applications.

\section{SUMMARY}

In this paper we have presented a detailed definition of a recently defined class of nonautonomous oscillatory systems, named chronotaxic ones [18]. Their defining property is the ability to sustain stability in the time-dependent amplitude and frequency of oscillations under continuous external perturbation. This ability stems from a driven (time-dependent) steady state which moves along the attracting cycle in phase space. The definitions of chronotaxic systems and chronotaxic limit cycles were extended by taking into account time-dependent and stable amplitudes. A wide area of potential applications for chronotaxic systems was discussed, and models with given time-dependent steady states were also presented. These may be especially useful in robotics for the design of oscillators with given properties. 
As discussed in Ref. [18], the properties of chronotaxic systems allow a reduction in the complexity of the full dynamics of these systems, making it possible to separate the motion of a driven steady state from dynamics that are contaminated by noise. Therefore, the development of corresponding methods for inverse problem methods will be of great importance. From a theoretical point of view, the general theory of chronotaxic systems in the case of entangled amplitude and phase dynamics remains an open question. One can expect that the recognition of chronotaxic systems will initiate intensive research in the area of nonautonomous oscillatory dynamical systems with stable dynamics.

\section{ACKNOWLEDGMENTS}

Our grateful thanks are due to J. Gratus, M. Horvat, D. Iatsenko, P. V. E. McClintock, and M. Rasmussen for valuable discussions, and V. Jirsa, A. Spiegler, and T. Proix for drawing our attention to Ref. [22], and anonymous reviewers for their helpful comments. This work was supported by the Engineering and Physical Sciences Research Council (UK) (Grant No. EP/100999X1).

\section{APPENDIX: ANALYTIC DERIVATION OF A TIME-DEPENDENT STEADY STATE}

Consider the system of equations:

$$
\dot{\alpha}_{\mathbf{p}}=\omega_{0}(t), \quad \dot{\alpha}_{\mathbf{x}}=\varepsilon \omega_{0}(t) \sin \left[\alpha_{\mathbf{x}}-\alpha_{\mathbf{p}}(t)\right],
$$

where $\alpha_{\mathbf{p}} \in R, \alpha_{\mathbf{x}} \in R$, and the frequency is assumed to be either positive $\omega_{0}(t)>0$ or negative $\omega_{0}(t)<0$ for all times $t$, and $\varepsilon \geqslant 1$. Using the new variable $\psi$ which is determined as $\psi=\alpha_{\mathbf{x}}-\alpha_{\mathbf{p}}$, the lower equation of (A1) can be rewritten as

$$
\dot{\psi}=\omega_{0}(t)(\varepsilon \sin \psi-1) .
$$

Solutions of this equation move from the vicinity of an unstable fixed point $\psi_{1}$, determined by $\dot{\psi}=0$ and $\frac{\partial \dot{\psi}}{\partial \psi}>0$, toward a stable fixed point $\psi_{2}$, determined by $\dot{\psi}=0$ and $\frac{\partial \dot{\psi}}{\partial \psi}<0$. When $\varepsilon>1$ Eq. (A2) always has a pair of stable and unstable fixed points. When $\varepsilon=1$ there is only one fixed point, and it is semistable.

Equation (A2) can be integrated, and solutions $\psi\left(t, t_{0}, \psi_{0}\right)$ can be found explicitly; here the initial condition is $\psi\left(t_{0}, t_{0}, \psi_{0}\right)=\psi_{0}$. By considering the limit $t_{0} \rightarrow-\infty$ one finds that all solutions $\psi\left(t, t_{0}, \psi_{0}\right)$ approach a single solution $\psi^{A}(t)$. Therefore the solution $\psi^{A}(t)$ is identified as a pullback attractor. Simultaneously, all points converge to $\psi^{A}(t)$ when $t \rightarrow+\infty$; therefore $\psi^{A}(t)$ is a time-dependent steady state. Taking into account the definition of $\psi$, the time-dependent steady state for the phase $\alpha_{\mathbf{x}}$ is given by

$$
\alpha_{\mathbf{x}}^{A}(t)=\alpha_{\mathbf{p}}(t)+\psi^{A}(t) .
$$

Here $\psi^{A}(t)$ is found in cases of positive frequency only as

$$
\psi^{A}(t)=\pi-\arcsin \frac{1}{\varepsilon}+2 \pi n, \quad \omega_{0}(t)>0 \text { for all times } t,
$$

or negative frequency only as

$$
\psi^{A}(t)=\arcsin \frac{1}{\varepsilon}+2 \pi n, \quad \omega_{0}(t)<0 \text { for all times } t,
$$

where $n$ is arbitrary integer number $n \in \mathbb{Z}$. These results are obtained by the integration of Eq. (A2):

$$
\int_{\psi\left(t_{0}\right)}^{\psi(t)} \frac{d \psi}{\varepsilon \sin \psi-1}=\int_{t_{0}}^{t} \omega_{0}\left(t^{\prime}\right) d t^{\prime}
$$

where it is assumed that $\varepsilon \sin \psi\left(t_{0}\right)-1 \neq 0$. The integration results in

$$
\begin{aligned}
& \frac{\tan \frac{\psi(t)}{2}-\varepsilon-\sqrt{\varepsilon^{2}-1}}{\tan \frac{\psi(t)}{2}-\varepsilon+\sqrt{\varepsilon^{2}-1}} \\
& \quad=\frac{\tan \frac{\psi\left(t_{0}\right)}{2}-\varepsilon-\sqrt{\varepsilon^{2}-1}}{\tan \frac{\psi\left(t_{0}\right)}{2}-\varepsilon+\sqrt{\varepsilon^{2}-1}} \exp \left[-\sqrt{\varepsilon^{2}-1} \int_{t_{0}}^{t} \omega_{0}\left(t^{\prime}\right) d t^{\prime}\right] .
\end{aligned}
$$

Taking into account that, according to our assumption, the denominators are nonzero in the above expression, one finds that the right-hand side of the equation equals zero when $\omega_{0}(t)>0$ and $t_{0} \rightarrow-\infty$. Therefore the left-hand side of the equation must be zero too, which is possible if

$$
\psi(t)=\pi-\arcsin \frac{1}{\varepsilon}+2 \pi n,
$$

thus leading to the solution (A4). Similarly, the solution (A5) can be found for a negative frequency $\omega_{0}(t)$.

In the general case, when $|\varepsilon| \geqslant 1$, and for either $\omega_{0}(t)>0$ or $\omega_{0}(t)<0$ for all $t$ one obtains

$$
\begin{aligned}
\alpha_{\mathbf{x}}^{A}(t)= & \alpha_{\mathbf{p}}(t)-\operatorname{sign}\left[\omega_{0}(t)\right] \arcsin (1 /|\varepsilon|) \\
& +\frac{\pi}{2}\left\{1+\operatorname{sign}\left[\varepsilon \omega_{0}(t)\right]\right\}+2 \pi n .
\end{aligned}
$$

Using (A9), the equation for the dynamics of $\alpha_{\mathbf{x}}$ in (A1) can be rewritten in terms of a time-dependent steady state $\alpha_{\mathbf{x}}^{A}$ :

$$
\begin{aligned}
\dot{\alpha}_{\mathbf{x}}= & \varepsilon \dot{\alpha}_{\mathbf{x}}^{A}(t) \sin \left(\alpha_{\mathbf{x}}-\alpha_{\mathbf{x}}^{A}(t)+\operatorname{sign}\left[\omega_{0}(t)\right] \arcsin (1 /|\varepsilon|)\right. \\
& \left.-\frac{\pi}{2}\left\{1+\operatorname{sign}\left[\varepsilon \omega_{0}(t)\right]\right\}\right) .
\end{aligned}
$$

Equation (32) refers to the case when $\varepsilon \geqslant 1$ and $\omega_{0}(t)>0$.
[1] A. E. Motter, S. A. Myers, M. Anghel, and T. Nishikawa, Nat. Phys. 9, 191 (2013).

[2] M. Hirota, M. Holmgren, E. H. V. Nes, and M. Scheffer, Science 334, 232 (2011).
[3] B. O’Rourke, B. M. Ramza, and E. Marban, Science 265, 962 (1994).

[4] F. A. Chandra, G. Buzi, and J. C. Doyle, Science 333, 187 (2011). 
[5] K. Friston, NeuroImage 5, 164 (1997).

[6] A. Volterra and J. Meldolesi, Nat. Rev. Neuro. 6, 626 (2005).

[7] J. A. Patz, D. Campbell-Lendrum, T. Holloway, and J. A. Foley, Nature (London) 438, 310 (2005).

[8] P. E. Kloeden and M. Rasmussen, Nonautonomous Dynamical Systems (American Mathematical Society, Providence, RI, 2011).

[9] M. I. Vishik and V. V. Chepyzhov, Math. Notes 51, 622 (1992).

[10] H. Crauel and F. Flandoli, Prob. Theory Rel. Fields 100, 365 (1994).

[11] F. J. Romeiras, C. Grebogi, and E. Ott, Phys. Rev. A 41, 784 (1990).

[12] C. S. Bretherton, M. Widmann, V. P. Dymnikov, J. M. Wallace, and I. Blade, J. Climate 12, 1990 (1999).

[13] M. D. Chekroun, E. Simonnet, and M. Ghil, Physica D 240, 1685 (2011).

[14] K. Saermark, Y. Ashkenazy, J. Levitan, and M. Lewkowicz, Physica A 236, 363 (1997).

[15] C. J. Stam, Clin. Neurophysiol. 116, 2266 (2005).

[16] P. M. C. de Oliveira, Theory Biosci. 120, 1 (2001).

[17] P. Ashwin, S. Wieczorek, R. Vitolo, and P. Cox, Phil. Trans. R. Soc. A 370, 1166 (2012).

[18] Y. F. Suprunenko, P. T. Clemson, and A. Stefanovska, Phys. Rev. Lett. 111, 024101 (2013).

[19] A. A. Andronov, A. A. Vitt, and S. E. Khaikin, The Theory of Oscillators (Pergamon Press, Oxford, 1966).

[20] Y. Kuramoto, Chemical Oscillations, Waves, and Turbulence (Springer-Verlag, Berlin, 1984).

[21] A. Pikovsky, M. Rosenblum, and J. Kurths, Synchronization-A Universal Concept in Nonlinear Sciences (Cambridge University Press, Cambridge, 2001).

[22] J. Buchli, L. Righetti, and A. J. Ijspeert, Biol. Cybern. 95, 645 (2006).

[23] P. E. Kloeden and V. S. Kozyakin, Dis. Cont. Dyn. Sys. 7, 883 (2001).

[24] P. E. Kloeden and T. Lorenz, J. Diff. Eqns. 253, 1422 (2012).

[25] H. Haken, Synergetics: An Introduction and Advanced Topics (Springer, Berlin, 2004).
[26] L. Kocarev and U. Parlitz, Phys. Rev. Lett. 76, 1816 (1996).

[27] P. E. Kloeden and V. S. Kozyakin, Dis. Cont. Dyn. Sys. 7, 883 (2001).

[28] R. He and P. G. Vaidya, Phys. Rev. A 46, 7387 (1992).

[29] L. M. Pecora and T. L. Carroll, Phys. Rev. Lett. 64, 821 (1990).

[30] L. M. Pecora and T. L. Carroll, Phys. Rev. A 44, 2374 (1991).

[31] A. Stefanovska and M. Bračič, Contemp. Phys. 40, 31 (1999).

[32] F. T. Kurz, M. A. Aon, B. O'Rourke, and A. A. Armoundas, Am. J. Physiol. Heart Circ. Physiol. 299, H1736 (2010).

[33] D. Rudrauf, A. Douiri, C. Kovach, J. P. Lachaux, D. Cosmelli, M. Chavez, C. Adam, B. Renault, J. Martinerie, and M. L. Van Quyen, NeuroImage 31, 209 (2006).

[34] K. Friston, Entropy 14, 2100 (2012).

[35] D. Konstantinov, A. D. Chepelianskii, and K. Kono, J. Phys. Soc. Jpn. 81, 093601 (2012).

[36] A. Stefanovska, M. Bračič Lotrič, S. Strle, and H. Haken, Physiol. Meas. 22, 535 (2001).

[37] A. Stefanovska, D. G. Luchinsky, and P. V. E. McClintock, Physiol. Meas. 22, 551 (2001).

[38] J. J. Suárez-Vargas, J. A. González, A. Stefanovska, and P. V. E. McClintock, Europhys. Lett. 85, 38008 (2009).

[39] T. Iadecola, D. Campbell, C. Chamon, C.-Y. Hou, R. Jackiw, S.-Y. Pi, and S. V. Kusminskiy, Phys. Rev. Lett. 110, 176603 (2013).

[40] J. Sonner and A. G. Green, Phys. Rev. Lett. 109, 091601 (2013).

[41] A. Stefanovska, M. Bračič, and H. D. Kvernmo, IEEE Trans. Bio. Med. Eng. 46, 1230 (1999).

[42] J. Jamšek, M. Paluš, and A. Stefanovska, Phys. Rev. E 81, 036207 (2010).

[43] L. W. Sheppard, A. Stefanovska, and P. V. E. McClintock, Phys. Rev. E 83, 016206 (2011).

[44] L. W. Sheppard, A. Stefanovska, and P. V. E. McClintock, Phys. Rev. E 85, 046205 (2012).

[45] T. Stankovski, A. Duggento, P. V. E. McClintock, and A. Stefanovska, Phys. Rev. Lett. 109, 024101 (2012).

[46] A. Duggento, T. Stankovski, P. V. E. McClintock, and A. Stefanovska, Phys. Rev. E 86, 061126 (2012).

[47] P. T. Clemson, Y. E. Suprunenko, T. Stankovski, and A. Stefanovska (unpublished). 